\title{
POISSON LIE GROUPS AND THEIR RELATIONS TO QUANTUM GROUPS
}

\author{
JANUSZ GRABOWSKI \\ Instytut Matematyki, Uniwersytet Warszawski \\ Banacha 2, 02-097 Warszawa, Poland \\ E-mail: jagrab@mimuw.edu.pl
}

\begin{abstract}
The notion of Poisson Lie group (sometimes called Poisson Drinfel'd group) was first introduced by Drinfel'd [1] and studied by Semenov-Tian-Shansky [7] to understand the Hamiltonian structure of the group of dressing transformations of a completely integrable system. The Poisson Lie groups play an important role in the mathematical theories of quantization and in nonlinear integrable equations.

The aim of our lecture is to point out the naturality of this notion and to present basic facts about Poisson Lie groups together with some relations to the recent work on quantum groups.
\end{abstract}

1. Poisson structures on manifolds. The basic mathematical structures of classical mechanics are the algebra $C^{\infty}(N)$ of all smooth functions on the phase space $N$ under ordinary multiplication and the Lie structure on $C^{\infty}(N)$ induced by the Poisson bracket $\{\cdot, \cdot\}$ defined by the symplectic form on $N$. The Poisson bracket is a biderivation of the associative algebra, i.e.

$$
\{f, g h\}=\{f, g\} h+g\{f, h\}
$$

and in so called canonical coordinates $(\mathbf{q}, \mathbf{p})=\left(q_{1}, \ldots, q_{n}, p_{1}, \ldots, p_{n}\right)$ has the form

$$
\{f, g\}=\sum_{i=1}^{n} \frac{\partial f}{\partial p_{i}} \frac{\partial g}{\partial q_{i}}-\frac{\partial f}{\partial q_{i}} \frac{\partial g}{\partial p_{i}},
$$

so the Hamilton equations

$$
\dot{\mathbf{p}}=-\frac{\partial H}{\partial \mathbf{q}}, \quad \dot{\mathbf{q}}=\frac{\partial H}{\partial \mathbf{p}}
$$

1991 Mathematics Subject Classification: 17B37, 58F05, 16W30, 81R50.

Lecture given at the Banach Center Colloquium on 7th January 1993.

Supported by the Erwin Schrödinger Institute for Mathematical Physics.

The paper is in final form and no version of it will be published elsewhere. 
may be written in the "Lax form":

$$
\dot{F}=\{H, F\} .
$$

We can forget about the symplectic form and consider a Lie bracket on $C^{\infty}(N)$ with the property (1.1).

(1.3) Definition. A Poisson bracket on an associative algebra is a Lie bracket which is a biderivation. A Poisson structure on a smooth manifold $N$ is a bivector field $P \in \Gamma\left(\Lambda^{2}(T N)\right)$ such that $\{f, g\}:=P(d f, d g)$ is a Poisson bracket on $C^{\infty}(N)$.

(1.4) R e m a r k. There is a one-one correspondence between Poisson brackets on $C^{\infty}(N)$ and Poisson structures on $N$ (which are those bivector fields $P$ which satisfy the equation $[P, P]=0$, where $[\cdot, \cdot]$ stands for the Schouten bracket of multivector fields).

For any $f \in C^{\infty}(N)$ the vector field $X_{f}:=i_{d f} P=\{f, \cdot\}$ is called the Hamiltonian vector field with Hamiltonian $f$ and the mapping $f \mapsto X_{f}$ is a homomorphism of the Lie algebra $C^{\infty}(N)$ with the Poisson bracket into the Lie algebra of vector fields.

(1.5) ExAmple. Every symplectic form induces a "classical" Poisson bracket with local form as described in (1.2). The corresponding Poisson structure has in the canonical coordinates the form

$$
P=\sum_{i=1}^{n} \frac{\partial}{\partial p_{i}} \wedge \frac{\partial}{\partial q_{i}}
$$

The "symplectic" Poisson brackets are exactly those which are non-degenerate in the sense that the mapping $\omega \mapsto P(\omega, \cdot)$ induces an isomorphism of the cotangent and tangent bundles. In particular, $N$ has to be even dimensional. One can show that every Poisson manifold admits a partition into symplectic submanifolds the orbits of Hamiltonian vector fields.

(1.6) ExAmple. Let $V$ be a Lie algebra over $\mathbf{R}$ with a basis $\left\{x_{1}, \ldots x_{n}\right\}$ and the bracket $[\cdot, \cdot]$. Set $N=V^{*}$ to be the dual space, so $x_{1}, \ldots, x_{n}$ can be regarded as smooth (in fact linear) functions on $N$ which form a coordinate system. It is easy to see that

$$
\{f, g\}:=\sum_{i, j=1}^{n}\left[x_{i}, x_{j}\right] \frac{\partial f}{\partial x_{i}} \frac{\partial g}{\partial x_{j}}=\sum_{i, j, k=1}^{n} c_{i j}^{k} x_{k} \frac{\partial f}{\partial x_{i}} \frac{\partial g}{\partial x_{j}},
$$

where $c_{i j}^{k}$ are the structure constants, is a Poisson bracket on $C^{\infty}\left(V^{*}\right)$ called Kostant-Souriau-Kirillov bracket. Linear functionals form a Lie subalgebra naturally identified with $(V,[\cdot, \cdot])$. The corresponding Kostant-Souriau-Kirillov Poisson structure is given by

$$
P=\sum_{i<j}\left[x_{i}, x_{j}\right] \frac{\partial}{\partial x_{i}} \wedge \frac{\partial}{\partial x_{j}}
$$


The symplectic leaves of this structure are exactly the orbits of the coadjoint representation of the corresponding Lie group.

Consider in particular the Lie algebra $s u(2)$ with the basis $(X, Y, H)$ and commutation relations

$$
[X, Y]=2 H, \quad[Y, H]=2 X, \quad[H, X]=2 Y .
$$

The Kostant-Souriau-Kirillov Poisson structure on the dual $s u(2)^{*}$ in chosen coordinates reads:

$$
P=2\left(H \frac{\partial}{\partial X} \wedge \frac{\partial}{\partial Y}+X \frac{\partial}{\partial Y} \wedge \frac{\partial}{\partial H}+Y \frac{\partial}{\partial H} \wedge \frac{\partial}{\partial X}\right)
$$

and symplectic leaves are 2-dimensional spheres in $s u(2)^{*} \simeq \mathbf{R}^{3}$ centred at 0 .

(1.7) Example. Let $N$ be the unit sphere in $\mathbf{R}^{4}$ with "global coordinates" $(a, b, x, y), a^{2}+b^{2}+x^{2}+y^{2}=1$. There is a unique Poisson bracket on $N$ satisfying

$$
\begin{aligned}
& \{x, a\}=-x b, \quad\{x, b\}=x a, \quad\{x, y\}=0, \\
& \{y, a\}=-y b, \quad\{y, b\}=y a, \quad\{b, a\}=x^{2}+y^{2} .
\end{aligned}
$$

The corresponding Poisson structure can be written in the form $P=A \wedge B$, where

and

$$
\begin{aligned}
& A=a C-\frac{\partial}{\partial a}, B=-b C+\frac{\partial}{\partial b}, \\
& C=a \frac{\partial}{\partial a}+b \frac{\partial}{\partial b}+x \frac{\partial}{\partial x}+y \frac{\partial}{\partial y} .
\end{aligned}
$$

Note that the vector fields $A, B$ are really tangent to the sphere and that $[B, A]=$ $a B+b A$.

If we identify this sphere with the group $S U(2)$ by

$$
(a, b, x, y) \mapsto\left(\begin{array}{cc}
\alpha & -\bar{\nu} \\
\nu & \bar{\alpha}
\end{array}\right),
$$

where $\alpha=a+i b, \nu=x+i y$, then $P$ can also be written in the form

$$
P=\left(x^{2}+y^{2}\right) \hat{X} \wedge \hat{Y}+(b y+a x) \hat{X} \wedge \hat{H}+(a y-b x) \hat{Y} \wedge \hat{H},
$$

where $\hat{X}, \hat{Y}, \hat{H}$ are the right-invariant vector fields on $S U(2)$ corresponding to the elements

$$
X=\left(\begin{array}{cc}
0 & -1 \\
1 & 0
\end{array}\right), \quad Y=\left(\begin{array}{cc}
0 & i \\
i & 0
\end{array}\right), \quad H=\left(\begin{array}{cc}
i & 0 \\
0 & i
\end{array}\right)
$$

of the Lie algebra $s u(2)$ of $S U(2)$.

(1.9) Definition. Let $\left(M, P_{M}\right)$ and $\left(N, P_{N}\right)$ be Poisson manifolds with Poisson brackets $\{\cdot, \cdot\}_{M}$ and $\{\cdot, \cdot\}_{N}$. A smooth mapping $\phi: N \longrightarrow M$ is called the Poisson mapping if the induced $\phi^{*}: C^{\infty}(M) \longrightarrow C^{\infty}(N)$ is a homomorphism of Poisson brackets:

$$
\{u \circ \phi, v \circ \phi\}_{N}=\{u, v\}_{N} \circ \phi, \quad u, v \in C^{\infty}(M) .
$$


The product Poisson bracket $\{\cdot, \cdot\}_{M \times N}$ on $M \times N$ is defined by

$$
\{u, v\}_{M \times N}(x, y)=\left\{u^{x}, v^{x}\right\}_{N}(y)+\left\{u^{y}, v^{y}\right\}_{M}(x),
$$

where clearly $u^{x}$ (resp. $u^{y}$ ) is the smooth function of $y \in N$ (resp. $x \in M$ ) obtained from $u \in C^{\infty}(M \times N)$ by keeping $x$ (resp. $y$ ) constant.

(1.10) R e m a r k. Note that the product Poisson structure is a bivector field $P_{M \times N}$ on $M \times N$ which under the projections of $M \times N$ onto $M$ and $N$ gives respectively $P_{M}$ and $P_{N}$. If we identify $C^{\infty}(M \times N)$ with an appropriate tensor product $C^{\infty}(M) \otimes C^{\infty}(N)$, we may also write the product Poisson bracket in the form:

$$
\left\{u_{1} \otimes u_{2}, v_{1} \otimes v_{2}\right\}_{M \times N}=\left\{u_{1}, v_{1}\right\}_{M} \otimes u_{2} v_{2}+u_{1} v_{1} \otimes\left\{u_{2}, v_{2}\right\}_{N} .
$$

2. Quantum spaces. Roughly speaking, we obtain quantum spaces deforming corresponding "dual" algebraic objects as for example the commutative algebras of a given class functions on the space (cf. Gel'fand-Najmark functor) can be deformed into noncommutative ones. This class may be arbitrarily chosen; Woronowicz [9] prefers to work with $C^{*}$-algebras what is more difficult, but fruitful, while Drinfel'd [2] works with purely algebraic version only.

To make the whole thing transparent, let us consider the very classical example of Woronowicz [9] for the group $S U(2)$ which topologically is a three dimensional sphere. We shall keep the notation of (1.7). The *-algebra $\mathcal{A}$ generated by matrix elements is dense in $C(S U(2))$ and can be characterized as the "maximal" unital commutative *-algebra generated by elements $\alpha, \nu$ (cf. (1.7)) and satisfying $\alpha^{*} \alpha+$ $\nu^{*} \nu=I$. Dropping the assumption about commutativity, Woronowicz proposed to consider the algebra $\mathcal{A}_{q}$ as the unital non-commutative *-algebra generated by $\alpha, \nu$ satisfying $\alpha^{*} \alpha+\nu^{*} \nu=I$ and additionally the commutation relations

$$
\begin{aligned}
\alpha \alpha^{*}-\alpha^{*} \alpha & =\left(2 q-q^{2}\right) \nu^{*} \nu, \quad \nu^{*} \nu-\nu \nu^{*}=0 \\
\nu \alpha-\alpha \nu & =q \nu \alpha, \quad \nu^{*} \alpha-\alpha \nu^{*}=q \nu^{*} \alpha .
\end{aligned}
$$

It is clear that for $q=0$ we get the previous commutative algebra, so $\mathcal{A}_{q}$ is a one-parameter deformation of $\mathcal{A}=\mathcal{A}_{0}$. The crucial point here is that the algebras $\mathcal{A}_{q}$ do not "collapse" for $1>q \geq 0$, i.e. the basis remains the same (for example $\left\{\nu^{* m} \nu^{n} \alpha^{k}, \nu^{* m} \nu^{n} \alpha^{* l}: k, m, n=0,1,2, \ldots\right.$ and $\left.l=1,2, \ldots\right\}$ is a basis in $\mathcal{A}_{q}$ for all $1>q \geq 0$ ), and we may consider the algebras $\mathcal{A}_{q}$ not as different objects, but as different multiplications $\circ_{q}$ on the same object, namely the algebra $\mathcal{A}$. This way we get so called formal deformation of $\mathcal{A}$, since the ${ }^{\circ}{ }_{q}$-product of elements $u, v \in \mathcal{A}$ reads

$$
u \circ_{q} v=u v+\sum_{n=1}^{\infty} q^{n} P_{n}(u, v) .
$$

For example, using the identification of $\mathcal{A}_{q}$ with $\mathcal{A}$ via the basis as above, we get

$$
\alpha \circ_{q} \nu=\nu \circ_{q} \alpha-\left(\nu \circ_{q} \alpha-\alpha \circ_{q} \nu\right)=\nu \alpha-q \nu \alpha
$$

and $\alpha \circ_{q} \alpha^{*}=\alpha^{*} \circ_{q} \alpha+\left(\alpha \circ_{q} \alpha^{*}-\alpha^{*} \circ_{q} \alpha\right)=\left(I-\nu^{*} \nu\right)+2 q \nu^{*} \nu-q^{2} \nu^{*} \nu$. 
Since the commutator bracket $[u, v]_{q}=u \circ_{q} v-v \circ_{q} u$, as for any associative algebra, is a biderivation, we get easily that

$$
\{u, v\}:=P_{1}(u, v)-P_{1}(v, u)
$$

is a Poisson bracket. Thus the first non-trivial term of a formal deformation gives us, after antisymmetrization, a Poisson bracket. Assuming that the term $P_{1}$ is given by a bidifferential operator, we always can, after a reparametrization, obtain $P_{1}$ antisymmetric. Indeed, consider a formal automorphism $u \mapsto B(u)=u+$ $q B_{1}(u)+o(q)$. A new but isomorphic product $\tilde{\circ}_{q}$ defined by $u \tilde{o}_{q} v:=B^{-1}\left(B(u) \circ_{q}\right.$ $B(v))$ has the form

$$
u \tilde{\circ}_{q} v=u v+q\left(P_{1}(u, v)+\delta B(u, v)\right)+o(q),
$$

where $\delta B(u, v)=u B(v)-B(u v)+B(u) v$ is the Hochschild coboundary of $B$. It is well known (cf. [8]) that the differentiable Hochschild cohomology of $C^{\infty}(N)$ is the space of multivector fields, so $P_{1}(u, v)=\{u, v\}+\delta B$ for some $B \in \operatorname{End}(\mathcal{A})$ and we can obtain $P_{1}$ being a Poisson bracket choosing the corresponding formal automorphism.

The idea of deformational quantization is now the following: the commutative associative algebra structure of $C^{\infty}(N)$ is only a room for the physics, a Poisson bracket (the first non-commutative term) gives us the classical mechanics, but what we really need is the quantum mechanics given by the "true" noncommutative product $\circ_{q}$ whose infinitesimal part delivers the Poisson bracket, i.e. classical mechanics (cf. [4]).

(2.3) ExAMPLE. From the commutation relations (2.1) defining the Woronowicz' quantum sphere we get easily the corresponding Poisson bracket which on matrix elements of $S U(2)$ has the form

$$
\begin{aligned}
& \{\alpha, \bar{\alpha}\}=2 \bar{\nu} \nu, \quad\{\nu, \bar{\nu}\}=0, \\
& \{\nu, \alpha\}=\nu \alpha, \quad\{\bar{\nu}, \alpha\}=\bar{\nu} \alpha .
\end{aligned}
$$

Passing to real functions $\alpha=a+i b, \quad \nu=x+i y$, we get a purely imaginary bracket with the imaginary part being exactly the Poisson bracket described in (1.7) (cf. also [3]).

3. Quantum groups. To get a quantum group, we must equip a quantum space, i.e. an associative algebra $A$, with additional structures: a coproduct $\Delta$ : $A \longrightarrow A \otimes A$ and so called counit and antipode. The tensor product (in an appropriate sense) $A \otimes A$ represents clearly functions on the product group $G \times G$. All objects, maps and properties are dual to those of the category of groups. For example, the coproduct is the dual of the group multiplication $G \times G \longrightarrow G$, counit to the embedding of the neutral element $e \hookrightarrow G$, etc. The coproduct is not only coassociative, but it is an algebra homomorphism (with the obvious associative algebra structure on $A \otimes A$ ) as the mapping between quantum spaces. What we get is usually called a Hopf algebra, so quantum groups are nothing 
but Hopf algebras which are deformations of Hopf algebras associated with group structures (e.g. the $C^{*}$-Hopf algebras of continuous functions on compact groups in Woronowicz' and the universal enveloping Hopf algebras of Lie algebras in Drinfel'd's approach). The obvious *-coproduct for the algebra $\mathcal{A}$ generated by matrix elements of the group $S U(2)$ is given by

$$
\Delta(\alpha)=\alpha \otimes \alpha-\nu^{*} \otimes \nu, \quad \Delta(\nu)=\nu \otimes \alpha+\alpha^{*} \otimes \nu .
$$

Woronowicz obtained his famous quantum $S U(2)$ group deforming this coproduct to $\Delta_{q}=\Delta+q D$, where $D(\alpha)=\nu^{*} \otimes \nu, D(\nu)=0$. This new coproduct is compatible with the deformed multiplication $\circ_{q}$, so we get a quantum group (if we do not care at the moment about counit and antipode). The compatibility means that

and hence

$$
\Delta_{q}\left(u \circ_{q} v\right)=\Delta_{q}(u) \circ_{q}^{\mathcal{A} \otimes \mathcal{A}} \Delta_{q}(v)
$$

$$
\Delta_{q}\left([u, v]_{q}\right)=\left[\Delta_{q}(u), \Delta_{q}(v)\right]_{q}^{\mathcal{A} \otimes \mathcal{A}}
$$

where $\circ_{q}^{\mathcal{A} \otimes \mathcal{A}}$ and $[\cdot, \cdot]_{q}^{\mathcal{A} \otimes \mathcal{A}}$ are the product and commutator bracket in $\mathcal{A} \otimes \mathcal{A}$. The identity (3.1) implies in particular

$$
\Delta(\{u, v\})=\{\Delta(u), \Delta(v)\}^{\mathcal{A} \otimes \mathcal{A}},
$$

where $\{\cdot, \cdot\}^{\mathcal{A} \otimes \mathcal{A}}$ is the product Poisson bracket on $\mathcal{A} \otimes \mathcal{A}$ (cf. (1.10)). Of course, all this works not only in the particular $S U(2)$ case, but in the general scheme as well. Writing (3.2) on the level of the group $G$, we get that the corresponding Poisson structure $P \in \Gamma\left(\Lambda^{2}(T G)\right)$ satisfies

$$
P\left(g_{1} g_{2}\right)=\left(L_{g_{1}}\right)_{*} P\left(g_{2}\right)+\left(R_{g_{2}}\right)_{*} P\left(g_{1}\right),
$$

where $\left(L_{g_{1}}\right)_{*}$ and $\left(R_{g_{2}}\right)_{*}$ are the left and right actions of $G$ on multivector fields. A multivector field $P$ satisfying (3.3) is called multiplicative.

(3.4) Definition. A Poisson Lie group is a Lie group $G$ equipped with a multiplicative Poisson structure.

We may say equivalently that a Poisson Lie group is a Lie group $G$ equipped with a Poisson structure such that the group multiplication $G \times G \longrightarrow G$ is a Poisson map (with the product Poisson structure on $G \times G$ ). It can be regarded as "infinitesimal" part of the quantum group being the deformation of $G$ (in the category of Hopf algebras, of course). Note also that Poisson Lie structures are never "symplectic", since they vanish at the neutral element.

(3.5) Another example. Consider a Lie algebra $V$ with a basis $\left(x_{1}, \ldots, x_{n}\right)$ (cf. (1.6)) and the universal enveloping algebra $U$ which is a Hopf algebra with the coproduct of the form $x \mapsto I \otimes x+x \otimes I$ for $x \in V$. Due to the CampbellBaker-Hausdorff theorem, $U$ is isomorphic as a vector space with the symmetric algebra over $V$, or in other words, with the algebra $A=\operatorname{Pol}\left(V^{*}\right)$ of polynomials on the dual space $V^{*}$. $A$ is therefore a quantum group being a deformation of the Abelian group $V^{*}$ (with respect to addition). The corresponding Poisson bracket 
$\{\cdot, \cdot\}$ on $V^{*}$ being the infinitesimal part of this deformation restricted to linear functionals from $V \subset U \simeq \operatorname{Pol}\left(V^{*}\right)$ coincides with the original bracket $[\cdot, \cdot]$ on $V$, so $\left\{x_{i}, x_{j}\right\}=\left[x_{i}, x_{j}\right]$ and we get the Poisson structure $P$ as in (1.6). It is clearly multiplicative, since $V^{*}$ acts trivially on tensor fields and $P$ is linear: $P\left(x^{*}+y^{*}\right)=P\left(x^{*}\right)+P\left(y^{*}\right)$. The universal enveloping algebra of a Lie algebra $V$ may be therefore regarded as a quantum group being the quantization of the dual space $V^{*}$ and the Kostant-Souriau-Kirillov bracket on it. The universal enveloping Hopf algebra can be deformed once more. For example the Drinfel'd-Jimbo's quantum $S L(2, \mathbf{C})$ group is the formal deformation of the universal enveloping of the Lie algebra $\operatorname{sl}(2, \mathbf{C})$ given by deformed commutators

$$
\left[H, X^{ \pm}\right]= \pm 2 X^{ \pm}, \quad\left[X^{+}, X^{-}\right]=\frac{2}{q} \operatorname{sh}\left(\frac{q H}{2}\right)
$$

and deformed coproduct

$$
\Delta\left(X^{ \pm}\right)=X^{ \pm} \otimes \exp \left(\frac{q H}{4}\right)+\exp \left(\frac{-q H}{4}\right) \otimes X^{ \pm}, \quad \Delta(H)=H \otimes I+I \otimes H,
$$

where $\left(X^{+}, X^{-}, H\right)$ is the standard basis of $\operatorname{sl}(2, \mathbf{C})$. For $q=0$ we get clearly the non-deformed universal enveloping Hopf algebra.

4. Poisson Lie groups, Lie bialgebras and dressing transformations. The standard references for this section are papers [1], [2], [5], [6], [7].

Since the tangent bundle of a Lie group $G$ can be canonically trivialized, we can regard any Poisson structure $P$ on $G$ as the mapping $\tilde{P}: G \longrightarrow \mathcal{G} \wedge \mathcal{G}$, for $\mathcal{G}$ being the Lie algebra of $G$, putting

$$
\tilde{P}(g)=\left(R_{g^{-1}}\right)_{*} P(g) .
$$

In other words, every Poisson structure may be written in the form $P=\sum_{i, j} r_{i j} \hat{X}_{i} \wedge \hat{X}_{j}$, where $\hat{X}_{i}$ are the right-invariant vector fields on $G$ associated with $x_{i} \in \mathcal{G}$ and $r_{i j}$ are smooth functions on $G$, so $\tilde{P}=\sum_{i, j} r_{i j} x_{i} \wedge x_{j}$. The multiplicativity of $P$ means that

$$
\tilde{P}\left(g_{1} g_{2}\right)=\left(A d_{g_{1}}\right) \tilde{P}\left(g_{2}\right)+\tilde{P}\left(g_{1}\right),
$$

i.e. $\tilde{P}$ is a 1-cocycle of $G$ relative to the adjoint representation of $G$ in $\mathcal{G} \wedge \mathcal{G}$. Passing to the intrinsic derivative $p=d_{e}(\tilde{P})$, we get $p: \mathcal{G} \longrightarrow \mathcal{G} \wedge \mathcal{G}$, which is a 1-cocycle of the Lie algebra $\mathcal{G}$ with coefficients in the adjoint representation of $\mathcal{G}$ in $\mathcal{G} \wedge \mathcal{G}$, i.e.

$$
p([x, y])=a d_{x}(p(y))-a d_{y}(p(x)) .
$$

The dual mapping is $p^{*}: \mathcal{G}^{*} \wedge \mathcal{G}^{*} \longrightarrow \mathcal{G}^{*}$. It is easy to see that for any smooth functions $u, v$ on $G$, we have

$$
p^{*}(d u(e), d v(e))=(d\{u, v\})(e)
$$

(the right hand term does not depend on the choice of $u$ and $v$, since the Lie Poisson bracket $\{\cdot, \cdot\}$ vanishes at $e$ !) and the Jacobi identity for the Poisson 
bracket implies now that $p^{*}$ is a Lie bracket itself.

(4.1) Definition. A Lie bialgebra is a Lie algebra $\mathcal{G}$ equipped with a 1-cocycle $p: \mathcal{G} \longrightarrow \mathcal{G} \wedge \mathcal{G}$ relative to the adjoint representation of $\mathcal{G}$ in $\mathcal{G} \wedge \mathcal{G}$ such that the dual mapping $p^{*}: \mathcal{G}^{*} \wedge \mathcal{G}^{*} \longrightarrow \mathcal{G}^{*}$ is a Lie bracket (i.e. satisfies the Jacobi identity).

Note that the notion of Lie bialgebra is self-dual, since if $(\mathcal{G}, p)$ is a Lie bialgebra then $\left(\mathcal{G}^{*}, q\right)$, where $q: \mathcal{G}^{*} \longrightarrow \mathcal{G}^{*} \wedge \mathcal{G}^{*}$ is dual to the Lie bracket $[\cdot, \cdot]: \mathcal{G} \wedge \mathcal{G} \longrightarrow \mathcal{G}$, is a Lie bialgebra (called the dual Lie bialgebra) as well.

(4.2) Theorem. Given a simple connected Lie group $G$, there is a one-one correspondence between Poisson Lie structures on $G$ and Lie bialgebra structures on $\mathcal{G}$ as described above.

(4.3) Definition. Let $(G, P)$ be a Poisson Lie group, $(\mathcal{G}, p)$ be the tangent Lie bialgebra, and $\left(\mathcal{G}^{*}, q\right)$ be the dual Lie bialgebra. The simple connected Poisson Lie group $\left(G^{*}, Q\right)$ whose tangent Lie bialgebra is $\left(\mathcal{G}^{*}, q\right)$ is called the dual Poisson Lie group.

(4.4) Definition. Let $(G, Q)$ be a Poisson Lie group, $(N, P)$ be a Poisson manifold, and let $\Phi: G \times N \longrightarrow N$ be an action of $G$ on $N$. We call this action a Poisson action if $\Phi$ is a Poisson map (with respect to the product Poisson structure on the product, of course).

(4.5) Remark. Note that if the Poisson structure on the group is trivial the Poisson action simply preserves the Poisson bracket on $N$. The major advantage of the above notion is that we allow also actions which do not preserve the Poisson structure, but describe a "hidden symmetry" and admit a reduction method.

(4.6) Theorem. Let $(G, P)$ be a Poisson Lie group, $(\mathcal{G}, p)$ be the tangent Lie bialgebra, $\left(\mathcal{G}^{*}, q\right)$ be the dual Lie bialgebra, and $\left(G^{*}, Q\right)$ be the dual Poisson Lie group. Then the mappings

$$
\begin{array}{ll} 
& \lambda: \mathcal{G}^{*} \ni \omega \mapsto i_{\hat{\omega}_{\lambda}} P \in \Gamma(T G) \\
\text { and } & \rho: \mathcal{G}^{*} \ni \omega \mapsto i_{\hat{\omega}_{\rho}} P \in \Gamma(T G),
\end{array}
$$

where $\hat{\omega}_{\lambda}$ and $\hat{\omega}_{\rho}$ are respectively the left-and right-invariant 1-forms on G generated by $\omega$, are Lie algebra homomorphisms of $\mathcal{G}^{*}$ into the Lie algebra of vector fields on $G$.

(4.7) Definition. Integrating $\lambda$ (resp. $\rho$ ) gives rise to a local (and global if the corresponding vector fields are complete) left (resp. right) dressing action of $G^{*}$ on $G$ and we say that this actions consist of left (resp. right) dressing transformations.

(4.8) Theorem. For a Poisson Lie group $(G, P)$, both the left and right dressing actions of $G^{*}$ on $G$ are Poisson actions.

Note that the dressing actions are in general really only Poisson and not preserving the Poisson structure on $G$. 
(4.9) Re mark. There is a way to understand the dressing actions of $G^{*}$ on $G$ (and vice versa) in the language of so called double groups (and Manin triples on the level of Lie algebras). One can namely construct (at least locally) a Lie group $H$ with $G^{*}$ and $G$ as closed subgroups such that the multiplication

$$
G \times G^{*} \ni\left(g, g^{*}\right) \mapsto g g^{*} \in H
$$

is a diffeomorphism. Having the projections $\pi_{G}: H \longrightarrow G$ and $\pi_{G^{*}}: H \longrightarrow G^{*}$, we get for example the right dressing action of $G^{*}$ on $G$ in the form

$$
G \times G^{*} \ni\left(g, g^{*}\right) \mapsto \pi_{G}\left(g g^{*}\right) \in G .
$$

Unfortunately, we have no place here to go into details.

(4.10) EXAMPLE. Differentiating (1.8) we get the Lie bialgebra structure on $s u(2)$ corresponding to the Poisson Lie structure of the Woronowicz' quantum $S U(2)$ group:

$$
p(H)=0, \quad p(X)=H \wedge X, \quad p(Y)=H \wedge Y .
$$

The dual Lie bialgebra is therefore the Lie algebra spanned by $\left(H^{*}, X^{*}, Y^{*}\right)$, with the commutation relations

$$
\left[X^{*}, Y^{*}\right]=0, \quad\left[H^{*}, X^{*}\right]=X^{*},\left[H^{*}, Y^{*}\right]=Y^{*},
$$

i.e. the Lie algebra $s b(2, \mathcal{C})$ of all $2 \times 2$ traceless upper triangular complex matrices with real diagonal elements, and the cobracket defined by:

$$
q\left(H^{*}\right)=2 X^{*} \wedge Y^{*}, q\left(X^{*}\right)=2 Y^{*} \wedge H^{*}, \quad q\left(Y^{*}\right)=2 X^{*} \wedge H^{*} .
$$

The corresponding double group $H$ is the group $S L(2, \mathcal{C})$ with the decomposition into subgroups $S U(2)$ and $S B(2, \mathcal{C})$ being the group of upper triangular matrices of $S L(2, \mathcal{C})$ with real positive diagonal elements.

(4.11) ExAmPLE. The analogous procedure for the Kostant-Souriau-Kirillov Poisson structure (cf. (1.6)) yields the Lie bialgebra structure on the commutative Lie algebra $V^{*}$ of the form:

$$
p\left(x_{k}^{*}\right)=\sum_{i<j} c_{i j}^{k} x_{i}^{*} \wedge x_{j}^{*}
$$

The dual Lie bialgebra is therefore the Lie algebra $V$ itself with the trivial cobracket and the dual Poisson Lie group is the simple connected Lie group $G$ with the Lie algebra $V$ and the trivial Poisson structure. The double Lie group is in this case the cotangent bundle $T^{*} G$ with $G$ and $V^{*}$ as closed subgroups. 


\section{Reference}

[1] V. G. Drinfel'd, Hamiltonian structures on Lie groups, Lie bialgebras and the geometric meaning of the classical Yang-Baxter equations, Soviet Math. Dokl. 27 (1983), 68-71.

[2] , Quantum groups, Proc. ICM, Berkeley, Vol. 1, Amer. Math. Soc. 1986, 789-820.

[3] J. Grabowski, Quantum SU(2) group of Woronowicz and Poisson structures, in: Differential Geometry and its Application, Proc. Conf. Brno 1989, Eds. J. Janyška and D. Krupka, World Scientific 1990, 313-322.

[4] - Abstract Jacobi and Poisson structures. Quantization and star-products, J. Geom. Phys. 9 (1992), 45-73.

[5] Y. Kosmann-Schwarzbach, Poisson-Drinfel'd groups, Publ. IRMA, Lille, Vol. 5, No. 12, 1987.

[6] J.-H. Lu and A. Weinstein, Poisson Lie groups, dressing transformations, and Bruhat decompositions, J. Differential Geom. 31 (1990), 501-526.

[7] M. A. Semenov-Tian-Shansky, Dressing transformations and Poisson Lie group actions, Publ. Res. Inst. Math. Sci. 21 (1985), 1237-1260.

[8] J. Vey, Déformation du crochet de Poisson sur une variété symplectique, Comment. Math. Helv. 50 (1975), 421-454.

[9] S. L. Woronowicz, Twisted SU(2) group. An example of a non-commutative differential calculus, Publ. Res. Inst. Math. Sci. 23 (1987), 117-181. 